\section{EBUS-guided mediastinal lung cancer staging: monitoring of quality standards improves performance}

This audit examined key performance indices related to endobronchial ultrasound (EBUS)guided mediastinal lung cancer staging before and after the introduction of defined quality standards, at four independent EBUS centres in one cancer network. Data from 642 procedures were prospectively collected and analysed. The introduction of standards was associated with a significant increase $(p<0.001)$ in sampling of key mediastinal lymph node stations ( $4 R, 4 \mathrm{~L}$ and 7) and a reduction in the variability of staging sensitivity between centres. These data reinforce the requirement for an appropriate regulatory framework for EBUS-transbronchial needle aspiration provision that includes quality assurance and performance monitoring.

\section{INTRODUCTION}

Endobronchial ultrasound-guided transbronchial needle aspiration (EBUS-TBNA) is recommended as the first-line investigation in patients with non-small cell lung cancer requiring pathological mediastinal staging. ${ }^{1-4}$ The expansion of EBUS services, which are primarily delivered by respiratory physicians, has been rapid across the UK. ${ }^{5}$ However, marked variation exists in how frequently EBUS is used $^{6}$ and there is a lack of widespread performance reporting. The European Society of Thoracic Surgeons (ESTS) has defined a set of standards for preoperative nodal staging using EBUS-TBNA. ${ }^{3}$ These standards mandate as a minimum: the visualisation of mediastinal lymph node (LN) stations 4R, 4L and 7, sampling of any $\mathrm{LN}$ measuring $>5 \mathrm{~mm}$ and sampling of at least three N2/3 nodal stations per patient. Furthermore, The British Thoracic Society (BTS) Quality Standards for Bronchoscopy set a target of $88 \%$ for sensitivity in nodal staging with EBUS-TBNA. ${ }^{7}$ Manchester Cancer is a large cancer network in the North West of England responsible for the diagnosis and treatment of over 2000 patients with lung cancer annually. This audit examined the performance of EBUS-guided mediastinal lung cancer staging before and after the adoption of ESTS minimum standards across this cancer network.

\section{MATERIALS AND METHODS}

Four independent centres were commissioned to deliver EBUS-TBNA for Manchester Cancer, centre 1 from 2010 and centres 2, 3 and 4 from 2012. Respiratory physicians performed all procedures, conscious sedation was used at all sites and only centre 4 had Rapid On Site Evaluation (ROSE). Quality standards for mediastinal staging were not initially defined. A standard database was installed at each site to collect procedure-related data prospectively. Staging procedure performance was audited before (1 January 2012 to 1 October 2013) and after (1 October 2013 to 1 October 2014) the introduction of ESTS minimum standards; purely diagnostic procedures were excluded. The location and number of all sampled LN stations was recorded in audit 1 (N1-3), but only N2/3 LNs in audit 2. Results of EBUS nodal staging, mediastinoscopy, intraoperative nodal sampling and 6 months of clinicalradiological follow-up were also recorded. EBUS-staging outcome was categorised as: true positive if $\mathrm{N} 2 / 3$ nodal metastases were correctly identified or true negative if correctly excluded and false negative if EBUS failed to identify the presence of N2/3 nodal metastases (including LN stations not accessible with EBUS). Key EBUS-staging performance indicators measured included: sensitivity to detect $\mathrm{N} 2 / 3$ nodal malignancy, negative predictive value (NPV), number of N2/3 LN stations sampled/procedure and prevalence of $\mathrm{N} 2 / 3$ nodal disease in the population staged.

\section{RESULTS}

A total of 642 staging EBUS procedures were submitted for analysis, 408 in the first and 234 in the second audit (table 1), outcome data was available in $97 \%$ $(n=623 / 642)$. Centre 3 submitted no outcome data and was therefore excluded from analysis. The number of staging procedures/centre was between 61 and 100 in the second audit. Mean number of LNs sampled ranged from 1.3 to $1.9 \mathrm{LNs}$ (N1-3) in audit 1 and 1.6 to 1.7 LNs (N2/ 3 ) in audit 2. The introduction of ESTS standards was associated with a significant increase in sampling of stations 4R (31\%$53 \%, \quad \mathrm{p}<0.001), \quad 4 \mathrm{~L} \quad(13 \%-29 \%$, $\mathrm{p}<0.001)$ and $7(31 \%-62 \%, \mathrm{p}<0.001)$; however, only $16 \%$ of procedures sampled the target of three or more N2/3 nodal stations. Sensitivity of EBUS staging across the network did not change $(85 \%$ and $86 \%$ for both audit periods), but the variability between centres reduced from 36\% (range 59\%-94\%) to 5\% (range 83\%$88 \%$ ) in audit 2 . Only centre 4 met the BTS sensitivity target of $88 \%$. The prevalence of $\mathrm{N} 2 / 3$ disease varied according to centre (46\%-71\%) and changed over time: reducing in centre 1 (55\%-46\%), but increasing in centres 2 and 4 (49\%$60 \%$ and $53 \%-71 \%$, respectively), reflecting differences in case selection. The overall NPV of EBUS staging was lower in audit 2; this was because NPV dropped from $92 \%$ to $68 \%$ at centre 4 .

\section{DISCUSSION}

The introduction and monitoring of quality standards, defining the requirements of EBUS-guided mediastinal lung cancer staging, was associated with a significant increase in mediastinal LN sampling (stations 4R, 4L and 7) across a large cancer network. This change may reflect a shift from targeted EBUS of enlarged or fludeoxyglucose avid LNs to a more systematic examination of the mediastinum. However, the target of three N2/ 3 nodal stations sampled per procedure was not reached, though the use of ROSE at centre 4 must be appreciated when interpreting these data as the identification of nodal malignancy at a single N3 station may negate the need for further sampling. The impact on sensitivity and NPV, key performance indices of EBUS staging, was mixed. Lack of data from centre 3 precludes a definitive conclusion for the network as a whole; however, sensitivity of EBUS-guided staging was $86 \%$, in the three centres where outcome data were available, just below the BTS benchmark of $88 \%$ and variability between sites had reduced. This contrasted with NPV, where performance was more inconsistent (68\%-89\%); this variability was associated with differences in the prevalence of $\mathrm{N} 2 / 3$ nodal disease between centres, suggesting further guidance on case selection may be warranted.

In conclusion, this audit shows that there is variability in the performance of EBUS-guided staging of lung cancer across a UK cancer network. The introduction of quality standards significantly improved mediastinal LN sampling, though it should be noted that one centre failed to provide any data. Overall, there is still clear room for improvement. A focus on sampling a minimum of three $\mathrm{N} 2 / 3$ stations per procedure may be important and perhaps could be facilitated by anaesthetic cover to allow deeper sedation or general anaesthesia.

The available data support the concerns of many, that rapid expansion of EBUS without robust monitoring of patient outcomes has the potential to expose patients with lung cancer to harm. We propose the development of outcomes-based training prior to independent practice and strong local commissioning to set appropriate 
Table 1 Audit results of performance indicators for staging EBUS across Manchester Cancer

\begin{tabular}{|c|c|c|c|c|c|c|c|c|}
\hline \multirow{2}{*}{$\begin{array}{l}\text { Site } \\
\text { Audit period }\end{array}$} & \multicolumn{2}{|l|}{ Network } & \multicolumn{2}{|l|}{ Centre 1} & \multicolumn{2}{|l|}{ Centre 2} & \multicolumn{2}{|l|}{ Centre 4} \\
\hline & 1 & 2 & 1 & 2 & 1 & 2 & 1 & 2 \\
\hline Staging EBUS & 408 & 234 & 334 & 100 & 42 & 61 & 32 & 73 \\
\hline Average LN/procedure & 1.9 & 1.7 & 1.9 & 1.7 & 1.3 & 1.6 & 1.8 & 1.7 \\
\hline \multicolumn{9}{|c|}{ No. of LN stations sampled per procedure } \\
\hline Missing & $1 \%$ & $0 \%$ & $0 \%$ & $0 \%$ & $5 \%$ & $0 \%$ & $3 \%$ & $0 \%$ \\
\hline 0 & $5 \%$ & $5 \%$ & $5 \%$ & $8 \%$ & $11 \%$ & $3 \%$ & $0 \%$ & $1 \%$ \\
\hline 1 & $28 \%$ & $39 \%$ & $24 \%$ & $29 \%$ & $48 \%$ & $49 \%$ & $50 \%$ & $44 \%$ \\
\hline 2 & $43 \%$ & $40 \%$ & $45 \%$ & $41 \%$ & $32 \%$ & $38 \%$ & $35 \%$ & $41 \%$ \\
\hline$\geq 3$ & $24 \%$ & $16 \%$ & $27 \%$ & $22 \%$ & $5 \%$ & $10 \%$ & $13 \%$ & $14 \%$ \\
\hline \multicolumn{9}{|l|}{ LN station sampled } \\
\hline $4 \mathrm{R}$ & $31 \%$ & $53 \%$ & $30 \%$ & $56 \%$ & $39 \%$ & $47 \%$ & $35 \%$ & $55 \%$ \\
\hline $4 \mathrm{~L}$ & $13 \%$ & $29 \%$ & $13 \%$ & $27 \%$ & $16 \%$ & $23 \%$ & $16 \%$ & $36 \%$ \\
\hline 7 & $31 \%$ & $62 \%$ & $31 \%$ & $78 \%$ & $40 \%$ & $66 \%$ & $19 \%$ & $38 \%$ \\
\hline True positive & 186 & 115 & 160 & 39 & 10 & 30 & 16 & 46 \\
\hline True negative & 179 & 91 & 149 & 54 & 18 & 24 & 12 & 13 \\
\hline False negative & 32 & 19 & 24 & 7 & 7 & 6 & 1 & 6 \\
\hline Missing data & 10 & 9 & 0 & 0 & 7 & 1 & 3 & 8 \\
\hline Sensitivity & $85 \%$ & $86 \%$ & $87 \%$ & $85 \%$ & $59 \%$ & $83 \%$ & $94 \%$ & $88 \%$ \\
\hline Sensitivity $95 \% \mathrm{Cl}(\%)$ & 80 to 90 & 78 to 91 & 81 to 91 & 71 to 93 & 33 to 81 & 67 to 93 & 69 to 100 & 76 to 95 \\
\hline NPV & $85 \%$ & $83 \%$ & $86 \%$ & $89 \%$ & $72 \%$ & $80 \%$ & $92 \%$ & $68 \%$ \\
\hline NPV 95\% Cl (\%) & 79 to 89 & 74 to 89 & 80 to 91 & 77 to 95 & 50 to 87 & 61 to 92 & 62 to 100 & 43 to 86 \\
\hline Overall prevalence of $\mathrm{N} 2 / 3$ & $52 \%$ & $55 \%$ & $55 \%$ & $46 \%$ & $49 \%$ & $60 \%$ & $53 \%$ & $71 \%$ \\
\hline
\end{tabular}

standards. We would also encourage the centralisation of staging EBUS-TBNA procedures to large volume centres and the attainment of BTS endorsement, as suggested by Sethi et al, ${ }^{8}$ to facilitate appropriate service delivery. Satisfactory outcomes should also be part of revalidation for EBUS operators and EBUS centres for the peer-review process.

Matthew Evison, ${ }^{1,2}$ Philip Crosbie, 1,2 Julie Martin, ${ }^{1}$ Rajesh Shah, ${ }^{3}$ Helen Doran, ${ }^{4}$ Zoe Borrill, ${ }^{5}$ Jennifer Hoyle, ${ }^{5}$ Durgesh Rana, ${ }^{6}$ Simon Bailey, ${ }^{7}$ Richard Booton ${ }^{1,2}$

${ }^{1}$ Manchester Thoracic Oncology Centre, University Hospital of South Manchester NHS Foundation Trust, Manchester, UK

${ }^{2}$ The Institute of Inflammation and Repair, The University of Manchester, Manchester, UK

${ }^{3}$ Department of Thoracic Surgery, University Hospital South Manchester, Manchester, UK

${ }^{4}$ Department of Pathology, University Hospital South Manchester, Manchester, UK

${ }^{5}$ Department of Respiratory Medicine, North

Manchester General Hospital, Pennine Acute NHS Trusts, Manchester, UK

${ }^{6}$ Department of Cytopathology, Central Manchester University Hospitals NHS Foundation Trust,

Manchester, UK
${ }^{7}$ Department of Respiratory Medicine, Central Manchester University Hospitals NHS Foundation Trust, Manchester, UK

Correspondence to Dr Matthew Evison, Manchester Thoracic Oncology Centre, University Hospital of South Manchester, Southmoor Road, Wythenshawe M23 9LT, UK; m.evison@nhs.net, matthew.evison@uhsm.nhs.uk

Competing interests None declared.

Provenance and peer review Not commissioned; externally peer reviewed.

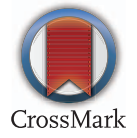

To cite Evison M, Crosbie P, Martin J, et al. Thorax 2016;71:762-763.

Received 27 February 2015

Revised 4 April 2016

Accepted 6 April 2016

Published Online First 4 May 2016

Thorax 2016;71:762-763.

doi:10.1136/thoraxjnl-2015-206985

\section{REFERENCES}

1 Silvestri GA, Gonzalez AV, Jantz MA, et al. Methods for staging non-small cell lung cancer: Diagnosis and management of lung cancer, 3rd ed: American College of Chest Physicians evidence-based clinical practice guidelines. Chest 2013;143(Suppl 5):e211S-50S.

2 NICE. Lung cancer: diagnosis and management. NICE guidelines [CG121], Apr 2011. https://www.nice.org. uk/guidance/cg121

3 De Leyn P, Dooms C, Kuzdzal J, et al. Revised ESTS guidelines for preoperative mediastinal lymph node staging for non-small-cell lung cancer. Eur J Cardiothoracic Surg 2014;45:787-98.

4 Vilmann P, Clementsen PF, Colella S, et al. Combined endobronchial and esophageal endosonography for the diagnosis and staging of lung cancer: European Society of Gastrointestinal Endoscopy (ESGE) Guideline, in cooperation with the European Respiratory Society (ERS) and the European Society of Thoracic Surgeons (ESTS). Endoscopy 2015;47:545-59.

5 Cusworth K, O'Dowd E, Hubbard R, et al. Variation in lung cancer resources and workload: results from the first national lung cancer organisational audit. Thorax2015;70:1001-3.

6 Cane P, Linklater K, Santis G, et al. The LungPath study: variation in the diagnostic and staging pathway for patients with lung cancer in England. Thorax 2016;71:291-3.

7 Du Rand IA, Barber PV, Goldring J, et al. British Thoracic Society guideline for advanced diagnostic and therapeutic flexible bronchoscopy in adults. Thorax 2011;66(Suppl 3):iii1-21.

8 Sethi T, Lim E, Peake M, et al. Improving care for patients with lung cancer in the UK. Thorax 2013;68:1181-5. 